Y. Miyahara

Nagoya Math. J.

Vol. 53 (1974), 157-170

\title{
OPTIMAL CONTROL OF ULTIMATELY BOUNDED STOCHASTIC PROCESSES
}

\author{
YOSHIO MIYAHARA
}

\section{§. Introduction}

We shall consider the optimal control for a system governed by a stochastic differential equation

$$
d X(t)=f(t, X(t), u(t, X(t))) d t+G(t, X(t), u(t, X(t))) d W(t),
$$

where $u(t, x)$ is an admissible control and $W(t)$ is a standard Wiener process. By an optimal control we mean a control which minimizes the cost and in addition makes the corresponding Markov process stable. For a concept of stability we require either the ultimate boundedness or the existence of a finite invariant measure of the Markov process. Accordingly our approach to the optimality problem is divided into two ways.

The first is as follows: Let $L(t, x, u)$ be a cost function and $\lambda$ be a positive constant. The cost is given by

$$
J_{t, x}^{*}[u]=\int_{t}^{\infty} e^{-\lambda(s-t)} M_{t, x} L(s, X(s), u(s, X(t))) d s,
$$

where $M_{t, x}$ denotes the expectation under the condition $X(t)=x$. An admissible control is a control such that the corresponding process is $p$-th ultimately bounded, that is, $\overline{\lim }_{s \rightarrow \infty} M_{t, x}\left|X^{u}(s)\right|^{p} \leqq K<\infty$ for any $(t, x) \in[0, \infty) \times \boldsymbol{R}^{n}$. An admissible control which attains the least cost is called an optimal control.

The second approach is limited to temporary homogeneous cases, where an admissible control is required that the corresponding process has an invariant probability measure $\mu$. An optimal control in this case is the admissible control which minimizes the cost given by

\footnotetext{
Received October 30, 1973.
} 


$$
J^{* *}[u]=\int_{R^{n}} L(x, u(x)) d \mu(x) .
$$

One may ask the relation between the above two concepts of optimality. However we have only been able to give a partial answer to this problem. (In § 3.)

In $\S 2$ we shall prove two theorems which give us sufficient conditions for the existence of optimal controls in the two senses respectively. The first one (Theorem 2.1*) shows that if the Bellman equation ((2.5) and (2.6)) has a nice solution, then the solution determines the optimal control in the first sense. The second theorem (Theorem $2.1^{* *}$ ) asserts that in the temporary homogeneous cases if an equation ((2.9) and (2.10)) has a nice solution, then the solution determines the optimal control in the second sense.

Applying these theorems, we shall consider an example in $\S 3$, which has been treated by W. M. Wonham [4]. We consider a temporary homogeneous linear system given by

$$
d X(t)=(A X(t)-B u(X(t))) d t+C d W_{1}(t)+G(X(t)) d W_{2}(t),
$$

where $G(x)=\sum_{i} x_{i} G_{i}$ and $A, B, C$ and $G_{i}$ are $n \times n, n \times m, n \times d_{1}$ and $n \times d_{2}$-dimensional constant matrices respectively, and where $W_{1}(t)$ and $W_{2}(t)$ are mutually independent standard Wiener processes. We assume that $(A, B)$ is controllable and that the cost function $L(x, u)$ is of the form

$$
L(x, u)=x^{\prime} M x+u^{\prime} N u, \quad x^{\prime} \text {; transpose of } x,
$$

where $M$ and $N$ are $n \times n$ and $m \times n$-dimensional symmetric positivedefinite constant matrices respectively. Then we can prove that all the conditions of Theorem $2.1^{*}$ and Theorem $2.1^{* *}$ are satisfied with $p=2$ and $\lambda>0$. Therefore we have optimal controls $u_{\lambda}^{*}(x), \lambda>0$, in the first sense and $u^{* *}(x)$ in the second sense. One of the interesting results is Theorem 3.2 which asserts that

$$
\lim _{\lambda \downarrow 0} u_{\lambda}^{*}(x)=u^{* *}(x) .
$$

This formula gives us an answer to the problem to make clear the relation between the two concepts of optimality.

The author wishes to thank Professor H. Kunita for his valuable advice. 


\section{§ 2. Optimality problems and criterion theorems}

We consider a stochastic process which is given by a stochastic differential equation

$$
d X(\mathrm{t})=f(t, X(t), u(t, X(t))) d t+G(t, X(t), u(t, X((t))) d W(t),
$$

where $f(t, x, u)$ is an $n$-dimensional vector-valued function defined on $[0, \infty) \times \boldsymbol{R}^{n} \times \boldsymbol{R}^{m}, \quad G(t, x, u) \quad$ is an $n \times d$-matrix valued function, $W(t)$ is a $d$-dimensional standard Wiener process and $u(t, x)$ is an admissible control. (The definition of admissible control will be given later.)

We will treat the optimality problem in two ways.

1. Optimality problem $(*)$

Suppose that we are given a continuous cost function $L(t, x, u)$ and two positive constants $p$ and $\lambda$. We say that an $m$-vector valued function $u(t, x)$ is admissible in the sense $(*)$ if it satisfies the following conditions (i) and (ii)*.

(i) $f(t, x, u(t, x))$ and $G(t, x, u(t, x))$ are continuous functions of $(t, x)$, and satisfy the assumptions $\left(\mathrm{A}_{1}\right)$ and $\left(\mathrm{A}_{2}\right)$;

$\left(\mathrm{A}_{1}\right)$ (Lipschitz continuity) there is a constant $K$ such that

$$
\begin{aligned}
|f(t, x, u(t, x))-f(t, y, u(t, y))| & \leqq K|x-y| \\
|G(t, x, u(t, x))-G(t, y, u(t, y))| \leqq K|x-y| & \text { for any }(t, x, y),
\end{aligned}
$$

$\left(\mathrm{A}_{2}\right)$

$$
\begin{aligned}
& |f(t, x, u(t, x))| \leqq K(|x|+1) \\
& |G(t, x, u(t, x))| \leq K(|x|+1),
\end{aligned}
$$

(ii)* the process $X^{u}(t)$ which is determined by (2.1) for given $u(t, x)$ is $p$-th ultimately bounded (see Y. Miyahara [2]).

We denote the set of all admissible controls by $\mathscr{U}^{*}$. For $u \in \mathscr{U}^{*}$ we define the cost by

$$
J_{t, x}^{*}[u]=\int_{t}^{\infty}\left\{e^{-\lambda(s-t)} M_{t, x} L\left(s, X^{u}(s), u\left(s, X^{u}(s)\right)\right)\right\} d s,
$$

where $(t, x)$ is the initial point and $M_{t, x}$ is the expectation under the condition $X^{u}(t)=x$.

DEFINITION 2.1. We call $u_{0}(t, x) \in \mathscr{U}^{*}$ an optimal control in the sense (*) if $J_{t, x}^{*}[u], u \in \mathscr{U}^{*}$, attains the minimum at $u_{0}$ for any $(t, x)$. The in- 
fimum $J^{*}[t, x]=\inf _{u \in \varkappa^{*}} J_{t, x}^{*}[u]$ is called the optimal cost.

2. Optimality problem (**)

We now consider temporary homogeneous cases, that is, we consider a system given by

$$
d X(t)=f(X(t), u(X(t))) d t+G(X(t), u(X(t))) d W(t) .
$$

Let a continuous cost function $L(x, u)$ and two constants $p(>0)$ and $\gamma$ be given. We assume here that $L(x, u) \geqq-c|x|^{p}-\beta$ for some positive constants $c$ and $\beta$. An $m$-dimensional vector valued function $u(x)$ is said to be admissible in the sense $(* *)$ if it satisfies;

(i) $f(x, u(x))$ and $G(x, u(x))$ are Lipschitz continuous,

(ii)** the corresponding process $X^{u}(t)$ has an invariant probability measure $\mu$ such that

$$
\int_{R^{n}}|x|^{p} d \mu(x)<\infty .
$$

We denote the set of all admissible controls in the sense $(* *)$ by $\mathscr{U}^{* *}$. The cost of $u \in \mathscr{U}^{* *}$ is defined by

$$
J^{* *}[u]=\inf _{\mu} J^{* *}[u, \mu],
$$

where

$$
J^{* *}[u, \mu]=\int_{R^{n}} L(x, u(x)) d \mu(x),
$$

and $\mu$ is an invariant measure of $X^{u}(t)$ given by (ii)**. We mention here that $J^{* *}[u, \mu]>-\infty$ by the assumption $L \geqq-c|x|^{p}-\beta$.

DEFinition 2.2. An admissible control $u_{0}(x) \in \mathscr{U}^{* *}$ is called an optimal control in the sense $(* *)$ if $J^{* *}[u], u \in \mathscr{U}^{* *}$, attains the minimum at $u_{0}$. The infimum $J^{* *}=\inf _{u \in \varkappa^{*}} J^{* *}[u]$ is called the optimal cost.

Remark 2.1. In temporary homogeneous cases, we know the relation $\mathscr{U}^{*} \subset \mathscr{U}^{* *}$. (Y. Miyahara [3])

We are now ready to state two criterion theorems.

THEOREM 2.1*. Assume that there exist a function $V(t, x)$ of $C^{(2)}$-class and an m-vector valued function $u_{0}(t, x)$ which satisfies the condition (i) of the definition of admissible control, and assume that the following conditions are satisfied; 


$$
\begin{gathered}
L(t, x, u) \geqq c_{1}|x|^{p}-\alpha, \\
0 \leq V(t, x) \leqq c_{2}|x|^{p}+\alpha, \\
\left|\frac{\partial V}{\partial x_{i}}\right|,\left|\frac{\partial^{2} V}{\partial x_{i} \partial x_{j}}\right| \leqq c_{3}\left(1+|x|^{\ell}\right), \quad i, j=1, \cdots, n, \\
-\lambda V(t, x)+\mathscr{L}_{u_{0}(t, x)} V(t, x)+L\left(t, x, u_{0}(t, x)\right)=0, \\
-\lambda V(t, x)+\mathscr{L}_{u} V(t, x)+L(t, x, u) \geqq 0, \\
\text { for any } u \in \boldsymbol{R}^{m},
\end{gathered}
$$

where $c_{1}, c_{2}, c_{3}, \alpha$ and $\ell$ are all positive constants and

$$
\begin{aligned}
\mathscr{L}_{u} V(t, x) & =\frac{\partial V}{\partial t}+\sum_{i} f_{i}(t, x, u) \frac{\partial V}{\partial x_{i}}+\frac{1}{2} \sum_{i, j} \sigma_{i, j}(t, x, u) \frac{\partial^{2} V}{\partial x_{i} \partial x_{j}}, \\
\left(\sigma_{i j}\right) & =G G^{*} .
\end{aligned}
$$

( $u$ can be a constant or a function of $t$ and $x$.) Then $u_{0}(t, x)$ is an optimal control in $\mathscr{U}^{*}$ and the function $V(t, x)$, being uniquely determined, coincides with the optimal cost $J^{*}[t, x]$. The process $X^{u_{0}}(t)$, corresponding to $u_{0}(t, x)$, is exponentially $p$-th ultimately bounded.

Remark 2.2. The function $V(t, x)$ turns out to be a Liapunov function of the exponentially $p$-th ultimately bounded process $X^{u_{0}}(t)$. This fact follows from the proof of Theorem 2.1*.

THEOREM 2.1**. Assume that there exist a function $V(x)$ of $C^{(2)}$-class, an admissible control $u_{0}(x) \in \mathscr{U}^{* *}$ and a constant $\gamma$ such that

$$
\begin{gathered}
|V(x)| \leqq c_{1}\left(|x|^{p}+1\right), \\
\left|\frac{\partial V}{\partial x_{i}}\right|,\left|\frac{\partial^{2} V}{\partial x_{i} \partial x_{j}}\right| \leqq c_{2}\left(1+|x|^{e}\right), \quad i, j=1, \cdots, n, \\
\mathscr{L}_{u_{0}} V(x)+L\left(x, u_{0}(x)\right)=\gamma, \\
\mathscr{L}_{u} V(x)+L(x, u) \geqq \gamma, \quad \text { for any } u \in \boldsymbol{R}^{m}, \\
L(x, u) \geqq-c_{3},
\end{gathered}
$$

where $c_{1}, c_{2}, c_{3}$, and $\ell$ are all positive constants. Then $u_{0}(x)$ is an optimal control in $\mathscr{U}^{* *}$ and the constant $\gamma$ coinsides with the optimal cost $J^{* *}$. The integral

$$
\int_{R^{n}} L\left(x, u_{0}(x)\right) d \nu(x)
$$


is equal to $\gamma$ for any invariant probability measure $\nu$ of $X^{u_{0}}$ which is given by the condition (ii)**.

CoRollary 2.1. Assume that there exist two functions $V(x)$ and $u_{0}(x)$, where $V(x)$ is of $C^{(2)}$-class and $u_{0}(x)$ satisfies the condition (i) of the definition of admissible control, and a constant $\gamma$. Further assume that $L(x, u), V(x), u_{0}(x)$ and $\gamma$ satisfy the conditions (2.7), (2.8), (2.9), (2.10) and

$$
\begin{aligned}
& L(x, u) \geqq c_{4}\left(|x|^{p}-\alpha_{1}\right), \\
& V(t, x) \geqq c_{5}\left(|x|^{p}-\alpha_{2}\right) .
\end{aligned}
$$

Then $u_{0}(x)$ belongs to $\mathscr{U}^{* *}$ and the conclusion of Theorem 2.1** holds.

Proof. It is sufficient to prove that $u_{0}(x) \in \mathscr{U}^{* *}$. From the assumptions of Corollary 2.1 all conditions of Theorem 3.1(B) in Y. Miyahara [2] are satisfied. Therefore $X^{u_{0}}(t)$ is exponentially $p$-th ultimately bounded, and it has a finite invariant measure by Y. Miyahara [3]. This proves that $u_{0}(x) \in \mathscr{U}^{* *}$.

Remark 2.3. These theorems are applicable in linear cases (see $\S 3$ ). But we have not known yet whether they are useful in non-linear cases too or not.

The rest of this section is devoted to give the proofs of the above two theorems.

\section{Proof of Theorem 2.1*}

Step 1. Choose any admissible control $u(t, x) \in \mathscr{U}^{*}$. By the condition (i) of the definition of admissible control and the assumptions (2.3) and (2.4) of $V(t, x)$, we are able to apply Dynkin-Ito formula to a function $e^{-\lambda t} V(t, x)$ to obtain

$$
\begin{aligned}
& e^{-\lambda s} M_{t, x} V\left(s, X^{u}(s)\right)-e^{-\lambda t} V(t, x) \\
& \quad=M_{t, x} \int_{t}^{s} \mathscr{L}_{u}\left\{e^{-\lambda \tau} V\left(\tau, X^{u}(\tau)\right)\right\} d \tau \\
& \quad \geqq-M_{t, x} \int_{t}^{s}\left\{e^{-\lambda \tau} L\left(\tau, X(\tau), u\left(\tau, X^{u}(\tau)\right)\right\} d \tau, \quad s>t \geqq 0,\right.
\end{aligned}
$$

where the last inequality comes from (2.6). If in particular we take $u(t, x)=u_{0}(t ; x)$, then we have an equality

$$
\begin{aligned}
& e^{-\lambda(s-t)} M_{t, x} V\left(s, X^{u_{0}}(s)\right) \\
& \quad=V(t, x)-M_{t, x} \int_{t}^{s}\left\{e^{-\lambda(\tau-t)} L\left(\tau, X^{u_{0}}(\tau), u_{0}(\tau, X(\tau))\right)\right\} d \tau .
\end{aligned}
$$


On the other hand it is proved that

$$
\varphi(s) \equiv e^{-\lambda s} M_{t, x} V\left(s, X^{u_{0}}(s)\right) \rightarrow 0 \quad(\text { as } s \rightarrow \infty) .
$$

In fact, (2.15) follows from

$$
\begin{aligned}
\frac{d \varphi(s)}{d t} & =-\frac{d}{d s}\left[\int_{t}^{s}\left\{e^{-\lambda \tau} M_{t, x} L\left(\tau, X^{u_{0}}(\tau), u_{0}(\tau)\right)\right\} d \tau \quad\right. \text { (by (2.14)) } \\
& =-e^{-\lambda s} M_{t, x} L\left(s, X^{u_{0}}(s), u_{0}(s)\right) \\
& \leqq-e^{-\lambda s} M_{t, x}\left\{c V\left(s, X^{u_{0}}(s)\right)-\beta\right\}, \quad \text { (by (2.2) and (2.3),) } \\
& =-c^{\prime} \varphi(s)+\beta e^{-\lambda s},
\end{aligned}
$$

where $c, c^{\prime}$ and $\beta$ are positive constants.

Two relations (2.14) and (2.15) prove that

$$
\begin{aligned}
V(t, x) & =M_{t, x} \int_{t}^{\infty}\left\{e^{-\lambda(\tau-t)} L\left(\tau, X^{u_{0}}(\tau), u_{0}(\tau)\right)\right\} d \tau \\
& =J_{t, x}^{*}\left[u_{0}\right] .
\end{aligned}
$$

Step 2. Let $u(t, x)$ be an admissible control. Then the corresponding process $X^{u}(t)$ is $p$-th ultimately bounded. We therefore have

$$
e^{-\lambda(s-t)} M_{t, x}\left|X^{u}(s)\right|^{p} \rightarrow 0, \quad(\text { as } s \rightarrow \infty),
$$

with this and (2.3) we easily see that (2.15) holds for any admissible control $u(t, x)$. We therefore conclude from (2.13) and (2.15), that

$$
J_{t, x}^{*}[u] \geqq V(t, x) .
$$

Step 3. We then prove that the process $X^{u_{0}}(t)$, corresponding to $u_{0}(t, x)$, is exponentially $p$-th ultimately bounded. It is sufficient to prove that all assumptions of Theorem 3.1(B) in [2] are satisfied. It is obvious that the assumptions (i), (ii)' and (iii) are satisfied. The only condition we have to prove is (ii), that is,

$$
V(t, x) \geqq c|x|^{p}-\beta
$$

for some positive constants $c$ and $\beta$. From Corollary 2.2 in [2 §2] we know thät

$$
M_{t, x}\left|X^{u_{0}}(s)\right|^{p} \geqq|x|^{p} e^{-k_{1}(s-t)}-k_{2}
$$

for some positive sonstants $k_{1}$ and $k_{2}$. Take a positive constant $T$, then we have the following formula by using (2.18), (2.2) and (2.20): 


$$
\begin{aligned}
V(t, x) & =\int_{t}^{\infty} e^{-\lambda(\tau-t)} M_{t, x} L\left(\tau, X^{u_{0}}(\tau), u_{0}(\tau)\right) d \tau \\
& \geqq \int_{t}^{t+T} e^{-\lambda(\tau-t)} M_{t, x} L\left(\tau, X^{u_{0}}(\tau), u_{0}(\tau)\right) d \tau-\alpha / \lambda \\
& \geqq \int_{t}^{t+T} e^{-\lambda(\tau-t)}\left(c_{1} M_{t, x}\left|X^{u_{0}}(\tau)\right|^{p}-\alpha\right) d \tau-\alpha / \lambda \\
& \geqq c_{4}|x|^{p}-c_{5} .
\end{aligned}
$$

This proves that $X^{u_{0}}(t)$ is exponentially $p$-th ultimately bounded, hence $u_{0} \in \mathscr{U}^{*}$.

(Q.E.D.)

Proof of Theorem 2.1**. Choose an admissible control $u(x)$ and consider $X^{u}(t)$. By the assumption (2.7) and (2.8) we are able to apply Dynkin-Ito formula to $V(x)$ to obtain

$$
M_{x} V\left(X^{u}(t)\right)-V(x)=\int_{0}^{t} M_{x} \mathscr{L}_{u} V\left(X^{u}(s)\right) d s .
$$

Let $\mu$ be an invariant measure of $X^{u}(t)$ given by (ii)** of the definition of admissible control. Then we have

$$
\begin{aligned}
\int_{R^{n}} & M_{x} V\left(X^{u}(t)\right) d \mu(x)-\int V(x) d \mu(x) \\
& =\int_{R^{n}} \int_{0}^{t} M_{x} \mathscr{L}_{u} V\left(X^{u}(s)\right) d s d \mu(x) \\
& \geqq \int_{R^{n}} \int_{0}^{t} M_{x}\left(-L\left(X^{u}(s), u\left(X^{u}(s)\right)+\gamma\right) d s d \mu(x) \quad\right. \text { (by (2.10)) } \\
& =-\int_{R^{n}} \int_{0}^{t} M_{x} L\left(X^{u}(s), u\left(X^{u}(s)\right)\right) d s d \mu(x)+\gamma t \\
& =-\int_{0}^{t} \int_{R^{n}} M_{x} L\left(X^{u}(s), u\left(X^{u}(s)\right)\right) d \mu(x) d s+r t \quad \text { (Fubini's theorem) } \\
& =-t \int_{R^{n}} L(x, u(x)) d \mu(x)+\gamma t .
\end{aligned}
$$

(The last integral may be $+\infty$ ).

The first formula in (2.23) is to be zero since $\mu$ is an invariant measure. Now we have

$$
\int_{R^{n}} L(x, u(x)) d \mu(x) \geqq \gamma
$$

If we set $u(x)=u_{0}(x)$, then we obtain, by (2.9), an equality in (2.24), namely 


$$
\int_{\boldsymbol{R}^{n}} L\left(x, u_{0}(x)\right) d \mu_{0}(x)=\gamma
$$

This completes the proof of Theorem 2.1**.

Remark 2.4. The condition (2.11) may be replaced by

$$
L(x, u) \geqq-c_{3}\left(|x|^{p}+1\right) .
$$

For we can use Fubini's theorem by the use of Corollary 2.1 of [2].

\section{§3. Relation between the problems $(*)$ and $(* *)$}

We shall illustrate a relation between the problems $(*)$ and $(* *)$ by showing an example due to W. M. Wonham [4].

Consider a system determined by

$$
d X(t)=(A X(t)-B u(X(t))) d t+C d W_{1}(t)+G(X(t)) d W_{2}(t),
$$

where $G(x)=\sum_{i=1}^{n} x_{i} G_{i}$ and $\mathrm{A}, \mathrm{B}, \mathrm{C}$ and $G_{i}$ are $n \times n, n \times m, n \times d_{1}$ and $n \times d_{2}$-dimensional constant matrices respectively, and where $W_{1}(t)$ and $W_{2}(t)$ are mutually independent standard Wiener processes. Only the case $p=2$ will be treated for simplicity. We assume that $(A, B)$ is controllable (see W. M. Wonham [5] for the definition) and that the cost function $L$ is of the form

$$
L(x, u)=x^{\prime} M x+u^{\prime} N u, \quad x^{\prime} \text {; transpose of } x,
$$

where $M$ and $N$ are $n \times n$ and $m \times n$-dimensional symmetric positivedefinite constant matrices respectively. This somewhat simple example will serve to illustrate a relation between the problems (*) and (**).

1. Problem $(*)$. Let $\lambda$ be a fixed positive constant, and put $\bar{A}(K)=$ $A-\frac{1}{2} \lambda E-B K$, where $K$ is an $m \times n$-matrix. We define an $n \times n$-matrix $\Gamma(P)$ by $\Gamma(P)_{k, 1}=\operatorname{trace}\left(G_{k}^{\prime} P G_{1}\right)$ for an $n \times n$-matrix $P$. Then $\Gamma(P)=\left(\Gamma(P)_{k, 1}\right)$ is a linear matrix-valued functional of $P$. It has a property that if $P$ is symmetric and non-negative definite so is $\Gamma(P)$. With these notations we state a theorem which is obtained as a consequence of Theorem 2.1*,

THEOREM 3.1.* The equation

$$
\begin{aligned}
& \left\{\bar{A}(K)^{\prime} P+P \bar{A}(K)+\left\{\Gamma(P)+M+K^{\prime} N K\right\}=0\right. \\
& K=N^{-1} B^{\prime} P
\end{aligned}
$$

has a unique symmetric positive solution $P^{\lambda}$. The function $u^{\lambda}(x)$ given by 


$$
u^{\lambda}(x)=K^{2} x, \quad \text { with } K^{\lambda}=N^{-1} B^{\prime} P^{\lambda},
$$

is an optimal control in $\mathscr{U}^{*}$ and the optimal cost is $J_{2}^{*}(x)=x^{\prime} P^{\lambda} x+$ $(1 / \lambda)$ trace $\left\{C^{\prime} P^{\lambda} C\right\}$. The corresponding process $X^{u \lambda}(t)$ is exponentially $2-n d$ ultimately bounded.

To prove this theorem we prepare several lemmas.

Lemma 3.1 (W. M. Wonham [5]). If $(A, B)$ is controllable, then for any $\left(\sigma_{1}, \cdots, \sigma_{n}\right) \in C^{n}$ there exists a matrix $K$ such that the eigen-values of $(A-B K)$ are $\left(\sigma_{1}, \cdots, \sigma_{n}\right)$.

Lemma 3.2 (W. M. Wonham [4, Lemma 3.1]). If a matrix $\bar{A}$ is sufficiently stable relative to the norm of $\Gamma(\cdot)$, then the equation

$$
\bar{A}^{\prime} P+P \bar{A}+\{\Gamma(P)+Q\}=0
$$

has a unique symmetric positive-definite solution $P$ for any symmetric positive matrix $Q$.

Proof of Theorem 3.1*. Using these lemmas, we are now able to constract a solution of (3.2) by the method of successive approximation. Given a matrix $K$, we consider a linear equation of $P$

$$
\bar{A}(K)^{\prime} P+P \bar{A}(K)+\left\{\Gamma(P)+M+K^{\prime} N K\right\}=0 .
$$

Step 1. Using Lemma 3.1 and Lemma 3.2, we can easily find a matrix $K_{0}$ such that the equation (3.4) has a unique symmetric positive solution $P_{0}$.

Step 2. Put $K_{1}=N^{-1} B^{\prime} P_{0}$ and denote the left side of (3.4) by $\Pi(P, K)$. Then we have $\Pi\left(P_{0}, K_{0}\right)=0$. From the definition of $K_{1}$, we see that

$$
x^{\prime} \Pi\left(P_{0}, K_{1}\right) x=\min _{K} x^{\prime} \Pi\left(P_{0}, K\right) x, \quad \text { for any } x .
$$

We therefore have an inequality $\Pi\left(P_{0}, K_{1}\right) \leqq \Pi\left(P_{0}, K_{0}\right)=0$, that is,

$$
\bar{A}\left(K_{1}\right)^{\prime} P_{0}+P_{0} \bar{A}\left(K_{1}\right)+\left\{\Gamma\left(P_{0}\right)+M+K_{1}^{\prime} N K_{1}\right\} \leqq 0 .
$$

Then the Liapunov's fundamental theorem proves that $\bar{A}\left(K_{1}\right)$ is stable. Therefore, the equation (3.4) is equivalent to

$$
P=\int_{0}^{\infty} e^{t \bar{A}\left(K_{1}\right)^{\prime}}\left[\Gamma(P)+M+K_{1}^{\prime} N K_{1}\right] e^{t \bar{A}\left(K_{1}\right)} d t
$$


It can be proved by the same way as Wonham [4] pp. 492 that this equation has a unique symmetric positive solution $P_{1}$ and $0<P_{1} \leq P_{0}$.

Step 3. We can inductively construct a sequence of solutions $\left(P_{n}, K_{n}\right)$ $n=0,1,2, \cdots$ such that $\Pi\left(P_{n}, K_{n}\right)=0$,

$$
P_{0} \geqq P_{1} \geqq \cdots \geqq P_{n} \geqq \cdots, \quad P_{n}>0, \quad K_{n}=N^{-1} B^{\prime} P_{n-1} .
$$

It is clear that there exist the limit matrices $P^{\lambda}$ and $K^{\lambda}$,

$$
P^{\lambda}=\lim _{n \rightarrow \infty} P_{n} \geqq 0, \quad K^{\lambda}=\lim _{n \rightarrow \infty} K_{n}=\lim _{n \rightarrow \infty} N^{-1} B^{\prime} P_{n}=N^{-1} B^{\prime} P,
$$

and that $\left(P^{\lambda}, K^{\lambda}\right)$ satisfies the equation (3.2).

Step 4. Put $V^{\lambda}(x)=x^{\prime} P^{\lambda} x+(1 / \lambda)$ trace $\left\{C^{\prime} P^{\lambda} C\right\}$ and $u_{0}^{\lambda}(x)=K^{\lambda} x$. Then it is easy to check that $V^{\lambda}(x)$ and $u_{0}^{\lambda}(x)$ satisfy all conditions of Theorem $2.1^{*}$. We therefore have proved that $u_{0}^{\lambda}(x)$ is an optimal control in $\mathscr{U}^{*}$ with the optimal cost $V^{\lambda}(x)$ and that the corresponding process $X^{u_{0}^{\lambda}}$ is exponentially ultimately bounded with a Liapunov function $V^{2}(x)$. The uniqueness of the solution of equation (3.2) is proved as follows: Let $\bar{P}$ be another solution of (3.2), then $\bar{u}(x)=N^{-1} B^{\prime} \bar{P}$ is also an optimal control and $\bar{V}(x)=x^{\prime} \bar{P} x+(1 / \lambda)$ trace $\left\{C^{\prime} \bar{P} C\right\}$ is to be an optimal cost. Hence we must have $\bar{P}=P^{\lambda}$. While the positivity of $P^{\lambda}$ is easily proved. In fact, since $V^{\lambda}(x)$ is a Liapunov function of $X^{u_{0}^{\lambda}}$, it follows that there are two positive constants $c$ and $c^{\prime}$ such that $V(x) \geqq c|x|^{2}-c^{\prime}$, which implies that $P^{\lambda}>0$. Thus we have proved theorem.

(Q.E.D.)

COROLLARY 3.1. If $\lambda_{1}>\lambda_{2}>0$, then $0<P^{\lambda_{1}} \leqq P^{\lambda_{2}}$, where both $P^{\lambda_{1}}$ and $P^{\lambda_{2}}$ are the solutions of (3.2).

Proof. Since $V^{\lambda}(x)$ is optimal cost and $L \geqq 0$, it is clear from the definition of cost that $V^{\lambda_{1}}(x) \leqq V^{\lambda_{2}}(x)$, that is,

$$
x^{\prime} P^{\lambda_{1}} x+\frac{1}{\lambda_{1}} \operatorname{tr}\left\{C^{\prime} P^{\lambda_{1}} C\right\} \leqq x^{\prime} P^{\lambda_{2}} x+\frac{1}{\lambda_{2}} \operatorname{tr}\left\{C^{\prime} P^{\lambda_{2}} C\right\}
$$

for any $x \in \boldsymbol{R}^{n}$.

Thus we have $P^{\lambda_{1}} \leqq P^{\lambda_{2}}$.

We then come to the investigation of the behavior of $P^{\lambda}$ when $\lambda \downarrow 0$.

LEMma 3.3. Let $P^{\lambda}$ be the solution of (3.2). Then the collection 
$\left\{P^{\wedge}\right\}, \lambda>0$, are bounded, that is, there exists a symmetric positive matrix $\bar{P}$ such that $0<P^{\lambda} \leqq \bar{P}$ for every $\lambda>0$.

Proof. Find a matrix $K_{0}$ for which the matrix

$$
\bar{A}\left(\lambda, K_{0}\right)=\left(A-\frac{\lambda}{2} E-B K_{0}\right), \quad \lambda \geqq 0,
$$

is stable enough to guarantee the existence of a unique positive solution $P_{0}^{\lambda}$ of (3.4) for every $\lambda \geqq 0$. This is possible by Lemma 3.1 and Lemma 3.2. We now prove that $P_{0}^{\lambda} \leqq P_{0}^{0}$ for any $\lambda>0$. As in W. M. Wonham [4, Lemma 3.1], the solution (3.4) $\lambda, \lambda \geqq 0$, can be obtained as follows:

$$
\begin{aligned}
& R^{\lambda} \equiv P_{0}^{\lambda}(1)=\int_{0}^{\infty} e^{t \bar{A}\left(\lambda, K_{0}\right)^{\prime}}\left[M+K_{0}^{\prime} N K_{0}\right] e^{t \bar{A}\left(\lambda, K_{0}\right)} d t, \\
& P_{0}^{\lambda}(2)=R^{\lambda}+T_{\lambda}\left(P_{0}^{\lambda}(1)\right),
\end{aligned}
$$

where

$$
\begin{aligned}
& \quad T_{\lambda}(P)=\int_{0}^{\infty} e^{t A\left(\lambda, K_{0}\right)^{\prime}} \Gamma(P) e^{t A\left(\lambda, K_{0}\right)} d t, \\
& \ldots \\
& P_{0}^{\lambda}(n)=R^{\lambda}+T_{\lambda}\left(P_{0}^{\lambda}(n-1)\right), \\
& P_{0}^{\lambda}(n) \longrightarrow P_{0}^{\lambda} \quad \text { as } n \rightarrow \infty .
\end{aligned}
$$

Since $\bar{A}\left(\lambda, K_{0}\right), \lambda>0$, is more stable than $\bar{A}\left(0, K_{0}\right)=\left(A-B K_{0}\right)$, we have

$$
\begin{aligned}
R^{\lambda}=P_{0}^{\lambda}(1) & =\int_{0}^{\infty} e^{t \bar{A}\left(\lambda, K_{0}\right)^{\prime}}\left[M+K_{0}^{\prime} N K_{0}\right] e^{t \bar{A}\left(\lambda, K_{0}\right)} d t \\
& \leqq \int_{0}^{\infty} e^{t\left(A-B K_{0}\right)^{\prime}}\left[M+K_{0}^{\prime} N K_{0}\right] e^{t\left(A-B K_{0}\right)} d t=P_{0}^{0}(1)=R^{0} \\
P_{0}^{\lambda}(2) & =R^{\lambda}+T_{\lambda}\left(P_{0}^{\lambda}(1)\right) \leqq R^{0}+T_{\lambda}\left(P_{0}^{0}(1)\right) \\
& \leqq R^{0}+T_{0}\left(P_{0}^{0}(1)\right)=P_{0}^{0}(2)
\end{aligned}
$$

and inductively $P_{0}^{\lambda}(n) \leqq P_{0}^{0}(n)$. Therefore we obtain

$$
P_{0}^{\lambda}=\lim _{n \rightarrow \infty} P_{0}^{\lambda}(n) \leqq \lim _{n \rightarrow \infty} P_{0}^{0}(n)=P_{0}^{0} .
$$

By Step 3 of the proof of Theorem 3.1* we know

$$
P_{n}^{\lambda} \downarrow P^{\lambda} \quad \text { as } n \rightarrow \infty .
$$

This fact and (3.7) prove that $P^{\lambda} \leqq P_{0}^{0}$.

As a consequence of Lemma 3.3 and Corollary 3.1 we know that

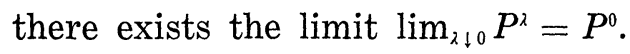


LEMMA 3.4. The positive matrix $P^{0}$, obtained above, is a solution of equation (3.2) with $\lambda=0$.

Proof. Because $P^{\lambda}$ is a solution of $(3.2)_{\lambda}$, the result is trivial by letting $\lambda \downarrow 0$.

(Q.E.D.)

THEOREM 3.2. The control $u^{0}(x)=K^{0} x=N^{-1} B^{\prime} P^{0} x=\lim _{\lambda \downarrow 0} K^{\lambda} x$ is an optimal control in $\mathscr{U}^{* *}$. The optimal cost $J^{* *}$ is equal to the trace $\left\{C^{\prime} P^{0} C\right\}$.

Proof. Since $P^{0}$ is a solution of (3.2) by Lemma 3.4, the result of this theorem can be obtained as a corollary of Theorem 3.1** which is to be proved in the following paragraph (Problem $(* *)$ ).

(Q.E.D.)

Remark 3.1. The corresponding process $X^{u^{2}}(t), \lambda>0$, tends to $X^{u^{0}}(t)$ in the sense of $L^{2}\left(\Omega \times[0, T], P_{x} \times d t\right)$-norm when $\lambda$ tends to 0 .

For proof we may proceed as follows: The solution of (3.1) is obtained by the method of successive approximation, where the convergence is uniform in $\lambda, 0 \leq \lambda \leq \lambda_{0}$. From this and the fact that

$$
\left|f\left(x, u^{\lambda}(x)\right)-f\left(x, u^{0}(x)\right)\right| \leq k(\lambda)|x|
$$

and $k(\lambda) \rightarrow 0(\lambda \rightarrow 0)$, we can prove that $X^{u^{\lambda}}(\cdot) \rightarrow X^{u^{0}}(\cdot)$ in the sense of $L^{2}\left(\Omega \times[0, T], P_{x} \times d t\right)$-norm.

2. Problem $(* *)$. If we consider the same example in the sense (**), then we have a theorem as a corollary of Theorem $2.1^{* *}$.

THEOREM 3.1.** If an equation

$$
\begin{aligned}
& (A-B K)^{\prime} P+P(A-B K)+\left\{\Gamma(P)+M+K^{\prime} N K\right\}=0 \\
& K=N^{-1} B^{\prime} P
\end{aligned}
$$

has a symmetric positive solution $P$, then $u_{0}(x)=N^{-1} B^{\prime} P x$ is an optimal control in $\mathscr{U}^{* *}$ and the optimal cost $J^{* *}$ is equal to the trace $\left\{C^{\prime} P C\right\}$.

Proof. If we put $V(x)=x^{\prime} P x, u_{0}(x)=N^{-1} B^{\prime} P x$ and $\gamma=\operatorname{trace}\left\{C^{\prime} P C\right\}$, then all the assumptions of Theorem $2.1^{* *}$ and Corollary 2.1 are satisfied. The method of the proof is similar to that of Theorem $3.1^{*}$, hence we omit the detail.

Remark 3.2. The method of the proof of Theorem $3.1^{* *}$ is essentially the same as that of W. M. Wonham [4]. 
COROLlaRY 3.2. Let $J_{2}^{*}(x)$ be the optimal cost of the problem (*) and $J^{* *}$ be the optimal cost of the problem (**) respectively. Then

$$
\lim _{\lambda \downarrow 0} \lambda J_{\lambda}^{*}(x)=J^{* *} \quad \text { for any } x \in \boldsymbol{R}^{n} \text {. }
$$

Proof. By Theorem 3.1* and Theorem 3.2, we know

$$
\begin{aligned}
\lambda J_{\lambda}^{*}(x) & =\lambda x^{\prime} P^{\lambda} x+\operatorname{trace}\left\{C P^{\lambda} C\right\}, \\
J^{* *} & =\operatorname{trace}\left\{C P^{0} C\right\} .
\end{aligned}
$$

Letting $\lambda \downarrow 0$, we have the desired result, because $\lim _{\lambda \downarrow 0} P^{\lambda}=P^{0}$ and $\left\{P^{\lambda}\right\}$, $\lambda \geq 0$, are bounded.

(Q.E.D.)

\section{BIBLIOGRAPHY}

[1] U. G. Haussmann; Optimal Stationary Control with State and Control Dependent Noise, SIAM J. Control, Vol. 9 No. 2 (1971) pp. 184-198.

[2] Y. Miyahara; Ultimate Boundedness of the Systems Governed by Stochastic Differential Equations, Nagoya Math. J. Vol. 47 (1972), pp. 111-144.

[3] —-; Invariant Measures of Ultimately Bounded Stochastic Process, Nagoya Math. J. Vol. 49 (1973) pp. 149-153.

[4] W. M. Wonham; Optimal Stationary Control of a Linear System with Statedependent Noise, SIAM J. Control, Vol. 5, No. 3 (1967).

[5] - ; On Pole assignment in Multi-input Controllable linear systems, IEEE Trans. Automatic Control, AC-12 (1967), pp. 660-665.

Shizuoka University 